\title{
Intensive Care Society Clinical Practice Free Paper Presentations
}

Tuesday, December 9, 2014

\section{CAPITAL SUITE ROOMS 2-4}

Protocolised procalcitonin testing in intensive care - a service development re-audit

PJ Antill, L Pareja-Cebrian, AH Rose

Who to admit and when to call the boss? A review of current training in assessing intensive care unit referrals and what influences discussion with senior colleagues

S Bakare, KC Tatham, DJP O'Callaghan

Can SIRS be used to identify patients with positive blood cultures?

D Brooks, A Smith, M Booth

Ceftazidime-induced neurotoxicity in renal failure

S Ganguly, M Sharafat

Treatment Escalation Plans - are they effective means of communication?

M Mercer, A Hulbert

Progressive reduction in the incidence of avoidable acquired pressure ulceration within an 18-bedded mixed general neurosciences level three ICU over 44 months: a quality improvement project

M Williamson, J Greenbaum, J Naisbitt

Predicting long-term mortality following admission to the intensive care unit in a UK Teaching Hospital: a scoring system based on the Sabadell score (abstract not available) $\checkmark$ Penston, D Lythgoe, T Astles, G Dempsey

An audit of clinical practice: oxygenation of critically ill patients in a university hospital general intensive care unit

BA Shuker, CR Bassford 


\section{Protocolised procalcitonin testing in intensive care - a service development re-audit}

PJ Antill, L Pareja-Cebrian, AH Rose

Pinderfields General Hospital, Wakefield, UK

Procalcitonin (PCT) has been hailed as a promising biomarker of sepsis, ${ }^{1}$ with developing interest in its uses in this age of multi-resistant organisms and increasing antibiotic burdens. Following a pre-implementation audit, a protocol for the use of procalcitonin testing in intensive care was introduced. ${ }^{2}$ A prospective re-audit was undertaken to assess adherence, and to review whether PCT testing in our practice improved antibiotic stewardship, inferred prognostication, or aided in the diagnosis and treatment of infection in intensive care, as has been suggested. ${ }^{3,4}$

Prospective data was collected from 50 patients undergoing a total of 111 PCT tests (range 1-7 tests per patient) within the intensive care units of the Trust between January and March 2014 (39 'medical', 10 'surgical', and one major burns patient). Each procalcitonin result was analysed against the protocol with regards to timing of the test and decisions made about antimicrobial therapy. Procalcitonin results were also correlated with microbiological, haematological and biochemical samples as well as patient mortality.

Peak procalcitonin levels were significantly greater in patients with positive blood cultures (median 13.47 [interquartile range 6.43-94.7]) as compared to those with no positive microbiology or with other positive microbiological samples $(1.24[0.58-3.98] \quad(p<0.01))$. This was despite some patients in the negative blood cultures group being known or suspected to have alternative causes of systemic inflammatory response syndromes (eg pancreatitis, burns, surgery). All patients with positive blood cultures had PCT $>0.99 \mathrm{ng} / \mathrm{mL}$, and no patient with PCT $<0.29 \mathrm{ng} / \mathrm{mL}$ had any positive microbiological samples. Our protocol "strongly encouraged" the use of antimicrobials if PCT $>1 \mathrm{ng} / \mathrm{mL}$ and "strongly discouraged" from use if PCT $<0.25 \mathrm{ng} / \mathrm{mL}$, thus supporting the implemented thresholds. ${ }^{2}$ We identified $52 \%$ of patients as having positive microbiological samples (compared to $31.7 \%$ in our previous audit), suggesting improved patient selection for testing. The protocol for antibiotic stewardship (to prescribe or not prescribe) with PCT testing was adhered to in $91.9 \%$ of cases. Peak procalcitonin results were higher in non-survivors (2.13 [0.74-37.44]) as compared to those surviving to hospital discharge (1.59 [0.54-5.32] $(\mathrm{p}=0.258)$ ).

A fine balance exists between rigid protocolisation and clinical freedom, with the aim of ensuring reproducibility in individual and departmental clinical practice. The implementation of procalcitonin testing in our intensive care department has shown it to share positive trends with microbiological samples and in-hospital mortality, demonstrating significant differences between those patients with blood-culture positive infections and those without. We found our protocol, which incorporates a degree of pre-test probability based on clinical judgement, to aid clinical decision-making regarding antibiotic stewardship despite the heterogeneity of our population. We found it particularly useful in the identification of patients with low PCT levels in whom antibiotics could be discontinued or omitted, ${ }^{2,3}$ such as diabetic ketoacidosis, asthma or burns, and when serial sampling was performed. Further work will assess both cost and quality implications of protocolised testing, incorporating not only the costs associated with testing and antimicrobial treatments themselves, but also considering the implications for medical, nursing and laboratory time, consumables, and the development of drug-resistant organisms and hospital-acquired infections.

\section{References}

1. Uzzan B, Cohen R, Nicholas P et al. Procalcitonin as a diagnostic test for sepsis in critically ill adults and after surgery or trauma: a systematic review and meta-analysis. Crit Care Med 2006:34:1996-2003.

2. Boudama L, Luyt CE, Tubach $\mathrm{F}$ et al. Use of procalcitonin to reduce patients' exposure to antibiotics in intensive care units (PRORATA trial): a multicentre randomised controlled trial. Lancet 2010;375:463-74

3. Kibe S, Adams K, Barlow G. Diagnostic and prognostic biomarkers of sepsis in critical care. J Antimicrob Chemother 2011:66:33-40.

4. Dellinger RP, Levy MM, Rhodes A et al. Surviving sepsis campaign: international guidelines for management of severe sepsis and septic shock: 2012. Crit Care Med 2013;41:580-637.

\section{Who to admit and when to call the boss? A review of current training in assessing intensive care unit referrals and what influences discussion with senior colleagues}

S Bakare* $^{*}$ KC Tatham* ${ }^{*}$ DJP O'Callaghan ${ }^{*}$

${ }^{*}$ Chelsea and Westminster Hospital, London UK. ${ }^{\dagger}$ Charing Cross Hospital, London, UK

Defining admission criteria for intensive care units (ICU) has attracted much interest, ${ }^{1}$ and while the overall consensus is that patients who are too sick or too well to benefit from an ICU admission should not be admitted, ${ }^{2}$ the practicality of identifying such patients is difficult in daily clinical practice. In fact, up to $33 \%$ of experienced ICU physicians will admit to inappropriate admissions secondary to clinical doubt. ${ }^{3}$

Furthermore it is likely that non-clinical factors such as bed shortages and pressure from referring clinicians, significantly influences decisions to admit patients to ICU. This, coupled with a lack of formal guidance and training on how to identify patients who would benefit from ICU admission, explains why $25 \%$ of ICU physicians admit to assessment errors leading to inappropriate ICU admissions. ${ }^{3}$ Inappropriate admissions place an avoidable strain on limited and expensive healthcare resources and can be emotionally challenging for patients, relatives and staff. We therefore sought to identify whether doctors covering ICUs had received any formal training in assessing patients referred to ICU and when and how they discussed these patients with their senior colleagues.

An anonymous questionnaire of 57 London deanery trainees (ranging from F2 to ST7) who covered ICUs was conducted to determine what formal training, if any, they had received on reviewing ICU referrals. We also sought to determine when referrals were discussed with senior colleagues.

Seventy-four per cent of trainees had not received any training on reviewing patients referred for ICU admission. Furthermore, 64\% of trainees wanted formal training on assessing ICU referalls. When asked about simple guidelines provided by their ICU team regarding key assessment parameters/triggers, 50\% felt such a tool would increase their confidence in contacting their consultant to discuss a referred patient.

Forty-five per cent of trainees always discuss ICU referrals with their consultants, with a further $39 \%$ doing so most of the time. Attitude of consultants (47\%) and access to beds (33\%) were among the highest reasons for discussing referrals with consultants.

Ninety-seven per cent of trainees who responded have been unsure at some point about the need for ICU admission of patients they reviewed, with $50 \%$ of trainees being unsure half of the time.

This data highlights the need for formal training in assessing ICU referrals. We have presented this data in our deanery, and talks are underway as to how formal teaching on assessing patients referred could be introduced at a regional level. A more structured approach, highlighting key parameters and triggers, and potentially consisiting of a strctured protocol, may increase trainee confidence and provide a degreee of consistency across institutions. Such a protocol may help identify when discussion with a senior colleague is appropriate and/or mandated.

\section{References}

1. Society of Critical Care Medicine Ethics Committee. Consensus statement on the triage of critically ill patients. JAMA 1994;271:1200-03.

2. Task Force of the American College of Critical Care Medicine. Guidelines for ICU admission, discharge, and triage. Crit Care Med 1999;27:633-38.

3. Giannini A, Consonni D. Physicians' perceptions and attitudes regarding inappropriate admissions and resource allocation in the intensive care setting. Br J Anaesth 2006;96: $57-62$.

\section{Can SIRS be used to identify patients with positive blood cultures?}

D Brooks*, A Smith*, M Booth

*University of Glasgow, UK. ${ }^{\star}$ Royal Infirmary, Glasgow, UK.

Reliably assessing the risk of bacteraemia before taking blood cultures 
would mean obtaining cultures in only patients at high risk of bacteraemia, thus reducing the number of negative cultures and the impact of falsepositive cultures. ${ }^{1}$ The systemic inflammatory response syndrome (SIRS) might be a sensitive indicator of the presence of bacteraemia. ${ }^{2}$ This study aimed to see if the number of SIRS criteria met can predict bacteraemia and thus whether SIRS can be used to reduce the number of negative cultures by identifying a cohort of patients unlikely to have bacteraemia in which blood cultures could be avoided.

Blood culture episodes drawn in Glasgow Royal Infirmary ICU between September 1st 2011 and December 31st 2013 were identified. SIRS criteria were recorded from time of phlebotomy using ICU information systems. A series of decision rules were created with each level of SIRS criteria in turn being set as the cut-off point to classify an episode as 'likely' or 'unlikely' to be bacteraemic. Predicted results were compared to actual results and cross-tabulations used to calculate sensitivity and specificity of each level of SIRS criteria. Logistic regression was used to calculate the odds of a positive culture with a given number of criteria.

Nine-hundred and fifty-five episodes had a documented time of phlebotomy (106 positive, 849 negative). Two SIRS criteria created 'unlikely to be positive' cohort of 181 (19\%) episodes. This rule had a sensitivity of $87 \%$, a specificity of $20 \%$ and would have prevented 167 (20\%) negative episodes (250 blood cultures) but missed 14 (13\%) positive episodes (22 blood cultures, 11 patients). Three SIRS criteria provided optimal trade-off between sensitivity (60\%) and specificity (50\%) and created an 'unlikely' cohort of 464 (49\%) episodes. This would have prevented 422 (49.7\%) negative episodes (670 blood cultures) with 42 ( $40 \%)$ positive episodes (74 blood cultures, 36 patients) missed. One and four criteria rules had a sensitivity of $95 \%$ and $17 \%$, prevented $38(4 \%)$ and $723(85 \%)$ negative episodes and misclassified five (4.7\%) and 88 (83\%) positive episodes respectively. No level of SIRS criteria significantly altered the odds of a blood culture being positive.

SIRS status is not suitable for assessing risk of bacteraemia - it results in a reduction in negative cultures but at all level of criteria the rule misclassified too many positive patients to be useful in clinical practice.

\section{References \\ 1. Coburn B, Morris AM, Tomlinson G et al. Does this adult patient with suspected bacteremia require blood cultures? JAMA 2012;308:502-11. \\ 2. Jones GR, Lowes JA. The systemic inflammatory response syndrome as a predictor of bacteraemia and outcome from sepsis. QJM 1996;89:515-22.}

\section{Ceftazidime-induced neurotoxicity in renal failure}

S Ganguly, M Sharafat

Leeds Teaching Hospital, Leeds, UK

A 70-year-old lady with end stage renal disease and ischaemic heart disease was admitted to the medical admission unit with symptoms of diarrhoea and vomiting. Her recovery was further complicated by hospital-acquired pneumonia and acute on chronic kidney disease.

According to local Trust protocol she was treated intravenously with two grams ceftazidime, three times a day. The following day there was a severe drop in her conscious level and one witnessed event of tonic-clonic seizure, which was resolved with lorazepam. A Glasgow Coma Scale of three was recorded with no eye opening, no verbal response and no motor response. Subsequently, she was transferred to intensive care where she was intubated and ventilated. Her head CT scan did not show any significant intracranial pathology, she had a lumbar puncture, which was normal. While being in the intensive care unit, she was started on continuous veno-venous haemofiltration for renal failure. An EEG was requested, which was reported to have a characteristic pattern of ceftazidime-induced neurotoxicity. Ceftazidime was stopped and continuous veno-venous haemofiltration was sustained to increase the clearance of ceftazidime for a few days during which she made a significant recovery. Within a few days she was discharged and sent back onto the ward.

Incidents of neurotoxicity with ceftazidime are low in clinical trials $(<1 \%)$. Characteristic EEG findings of ceftazidime-induced neurotoxicity demonstrates epileptic activity; that is, epileptiform discharges such as polyspike discharges, rhythmic slow waves, or irregular spikes or sharps, which initially can be confined to one region but may become more widespread, consequently spreading to both cerebral hemispheres. The main mechanism postulated for this kind of reaction is involvement of Gama Amino Butyric Acid A receptor inhibition. Predisposing factors for neurotoxicity with these antibiotics are patients receiving high doses, renal disease, blood-brain barrier damage and preexisting disease of the central nervous system. The reported clearance of ceftazidime during haemofiltration ranges from 4.2 to $24.0 \mathrm{~mL} / \mathrm{min}$.

We recommend that clinicians should have the necessary knowledge and awareness of the neurotoxic clinical manifestations, EEG findings, underlying mechanisms and predisposing factors. In particular, they need to know about renal diseases in order to help identify and treat this potentially lethal, but reversible complication of ceftazidime.

\section{Treatment Escalation Plans - are they effective means of communication?}

M Mercer, A Hulbert

South Devon Healthcare NHS Foundation Trust, Torquay, UK

In recent years Treatment Escalation Plans (TEPs) have been developed as an evolution of the Do Not Attempt Resuscitation (DNAR) forms. The intention is to broaden the documented medical plan beyond cardiopulmonary resuscitation. The TEP practice has been rolled out across Devon, UK, and is used in both the acute and community setting.

TEPs are often focused at, but not exclusive to, patients near the end of their lives and those likely to suffer a clinical deterioration. Medical management can present a significant challenge in these patients, especially when making decisions around escalation of care. This can range from deciding whether to admit to an acute hospital (if in the community), active ward-based management, admission to intensive care, or even or whether to opt for a palliative approach.

Treatment escalation planning concentrates on documenting patientcentred decisions and planning ahead in case of loss of capacity. The aim of this study was to assess whether the manner in which people die and the treatment they receive towards the end of life is consistent with the plan documented in their TEP form.

Fifty-two patients who had died in our hospital were identified. The patients were aged $>70$ years and from a five-week period spanning August and September 2013. Their notes were analysed for consistency or deviation away from the seven treatment selections present on the TEP form.

Of the 52 patients analysed, 46 patients had TEP forms in place. In six of the seven treatment arms recorded in the TEP form there were no deviations in terms of giving an undocumented treatment. The only single variance occurred where cardiopulmonary resuscitation (CPR) was attempted when the TEP documented it as being inappropriate. This was an out-of-hospital cardiac arrest in transit to our Trust, where the attending staffs were unaware of the TEP form. This study suggests the TEP process correlates well with the treatment patients receive at the end of life.

We conclude that the TEP is an effective means of communicating patients' care plans and this transcribes into real world treatment that is appropriate for individual patients.

\section{Progressive reduction in the incidence of avoidable acquired pressure ulceration within an 18-bedded mixed general neurosciences level three ICU over 44 months: a quality improvement project}

M Williamson, J Greenbaum, J Naisbitt

Salford Royal NHS Foundation Trust, UK

"If he has a bedsore, it's generally not the fault of the disease, but of the nursing" - Florence Nightingale, 1859 
Pressure ulceration is a common but often avoidable cause of significant patient morbidity and mortality. They are graded in severity from one to four and can be acquired in traditional pressure areas, such as the sacrum or heels, or discretely related to indwelling medical devices, eg nasogastric tubes.

Between January 2010 and 201153 of our patients acquired avoidable Grade 2, 3 or 4 pressure ulcers during their stay on the intensive care unit. In conjunction with an executive supported trust wide initiative, we embarked on a multidisciplinary clinical practice quality improvement intervention to reduce preventable harm.

Our objectives were to achieve a $50 \%$ reduction in the incidence of Grade 2 pressure sores and eliminate the incidence of Grade 3 and 4 pressure sores entirely.

The guiding team for this change in practice consisted of both senior nursing staff and consultants with the support of the trust QI department. We utilized the Model for Improvement methodology with multiple PlanDo-Study-Act cycles and continuous contemporaneous data collection. ${ }^{1}$ All pressure ulcers were individually verified and classified by tissue viability nursing staff independent of unit staff.

Our interventions included:

- Introduction of the SSKIN care bundle ${ }^{2}$

- Two-hourly patient repositioning

- Routine mandatory use of pressure-relieving NIMBUS ${ }^{\mathrm{TM}}$ mattress or rotational spinal beds

- The use of protocolised fixation methods for indwelling medical devices

- Adverse incident reporting and investigation by the ICU governance team

- MDT root cause analysis meetings for shared learning after each avoidable ulceration

- Mandatory educational training for all staff based on the aetiology of pressure ulceration and the SSKIN care bundle.

The results showed:

- January 2011-2012 ICU patients acquired 53 pressure ulcers (9.1 per 1000 bed days; 52.4 per 1000 admissions)

- January 2012-2013 ICU patients acquired 23 pressure ulcers (3.71 per 1000 bed days; 13.4 per 1000 admissions)

- January 2013-2014 ICU patients acquired 14 pressure ulcers (2.2 per 1000 bed days; 12.6 per 1000 admissions)

- From January-August 2014 ICU patients acquired three pressure ulcers. There were five traditional area-related pressure ulcers during the entirety of the project, 88 pressure ulcers were medical device related. There have been no pressure ulcers in ICU since March 2014.

We describe a successful clinical practice QI project to reduce the incidence of avoidable pressure ulceration within a Level 3 ICU. This work has resulted in a substantial reduction in patient harm and costs.

\section{References}

1. Langley GJ, Moen RD, Provost LP et al. The Improvement Guide. Second Edition: ISBN 978-0-470-19241-2

2. Whitlock J. SSKIN bundle: preventing pressure damage across the health-care community. Br J Community Nurs 2013: S32, S34-6, S38-9.

\section{An audit of clinical practice: oxygenation of critically ill patients in a university hospital general intensive care unit}

BA Shuker*, CR Bassford

*University of Warwick, Coventry, UK. "University Hospital Coventry, UK

Oxygen therapy is ubiquitous in the care of critically ill patients. The dangers of hypoxaemia are well described, however there is now a burgeoning body of evidence associating excessive arterial oxygen tensions (hyperoxaemia) with adverse patient outcomes. These include: increased mortality in chronic obstructive pulmonary disease; increased stroke severity and mortality; increased mortality after resuscitation from cardiac arrest; and increased overall mortality in ventilated critically ill patients. ${ }^{1-3}$

This audit aimed to investigate levels of oxygenation within a cohort of general intensive care admissions to determine the prevalence of potentially damaging levels of oxygenation.

Routinely collected records of arterial blood gas (ABG) samples were retrieved for all 2012 and 2013 admissions to a general intensive care unit at a university hospital. Venous, mixed venous, capillary, unlabelled samples, and samples missing oxygenation values $\left(\mathrm{FiO}_{2}, \mathrm{PaO}_{2}\right)$ were excluded.

The mean $\mathrm{PaO}_{2}$ from the duration of intensive care admission was calculated, and this was used to categorise admissions into three groups: hypoxaemia, normoxaemia, and hyperoxaemia. Hyperoxaemia was defined as $\mathrm{PaO}_{2} \geq 13.3 \mathrm{kPa}^{4}$ Normoxaemia was defined as neither hyperoxaemia nor hypoxaemia $\left(\mathrm{PaO}_{2}<8 \mathrm{kPa}\right)$.

Retrieval yielded 185,474 ABG records from 2,916 admissions, of which 79,051 ABG records met the inclusion criteria. The mean number of ABG samples collected per admission was 27.10 (SD 43.93), the minimum was $1(n=114)$, the maximum was 526 , and the mode was $4(n=226)$.

The grand mean $\mathrm{PaO}_{2}$ for all admissions was $13.80 \mathrm{kPa}$ (SD 3.368), the grand mean $\mathrm{FiO}_{2}$ and $\mathrm{SpO}_{2}$ were 0.42 (SD 0.16), and 97.16 (SD 3.76) respectively.

Almost half ( $n=1,411 ; 48.38 \%$ ) of admissions were in the hyperoxaemia group. Within the hyperoxaemia group $25 \%(\mathrm{n}=358)$ had a mean $\mathrm{PaO}_{2}$ of $\geq 17.0 \mathrm{kPa}$, and $5 \%(\mathrm{n}=70)$ had a mean $\mathrm{PaO}_{2}$ of $\geq 21.6 \mathrm{kPa}$. The median for the hyperoxaemia group was $15.10 \mathrm{kPa}$.

The normoxaemia group reflected just over half of admissions $(\mathrm{n}=1,477 ; 50.65 \%)$, and a small number of admissions were in the hypoxaemia group ( $\mathrm{n}=28 ; 0.096 \%)$.

In conclusion, almost half of admissions to a university hospital general intensive care unit over a two-year period had a mean arterial oxygen tension exceeding the normal range for the duration of their care. Given the potential for harm, oxygen therapy in critical care should be delivered with caution not excess.

Implications for clinical practice:

- Current oxygen therapy practice should be re-evaluated

- Oxygen therapy should target normoxaemia in all critically ill patients

- Clinicians should be vigilant for hyperoxaemia.

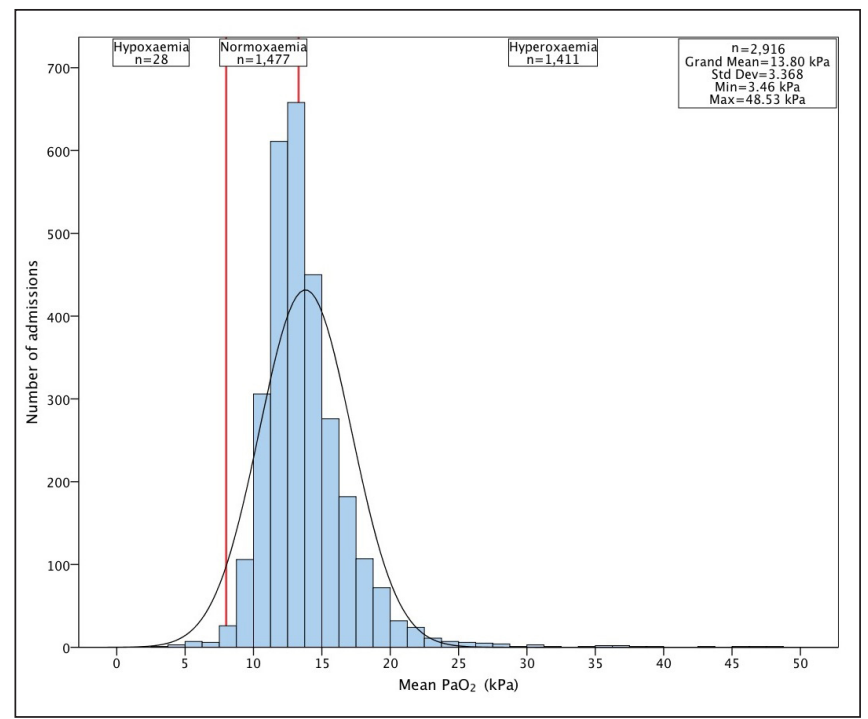

Figure 1. Mean oxygenation during intensive care stay.

\section{References}

1. Suzuki S, Eastwood GM, Bellomo R. A re-evaluation of oxygen therapy and hyperoxemia in critical care. In: Vincent JL (Ed.) Annual Update in Intensive Care and Emergency Medicine 2014. Springer International Publishing, 2014. p.81-91.

2. Rincon F, Kang J, Maltenfort M et al. Association between hyperoxia and mortality after stroke: a multicenter cohort study. Crit Care Med 2014:42:387-96.

3. de Jonge E, Peelen L, Keijzers PJ et al. Association between admin- istered oxygen, arterial partial oxygen pressure and mortality in mechanically ventilated intensive care unit patients. Crit Care 2008;12:R156.

4. Singer M, Webb A. Oxford Handbook of Critical Care, 3rd edn. Oxford: Oxford University Press, 2009. 